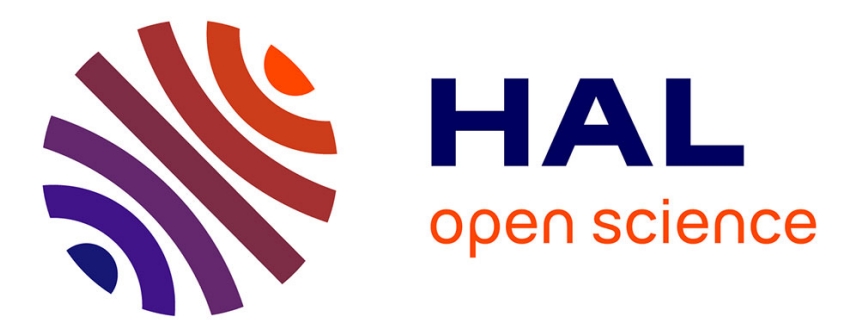

\title{
Optical calcium test for measurement of multiple permeation pathways in flexible organic optoelectronic encapsulation
}

Patrick Boldrighini, Aurélie Fauveau, Sandrine Thérias, Jean-Luc Gardette, Manuel Hidalgo, Stéphane Cros

\section{To cite this version:}

Patrick Boldrighini, Aurélie Fauveau, Sandrine Thérias, Jean-Luc Gardette, Manuel Hidalgo, et al.. Optical calcium test for measurement of multiple permeation pathways in flexible organic optoelectronic encapsulation. Review of Scientific Instruments, 2019, 90 (1), 10.1063/1.5021182 . hal03022935

\section{HAL Id: hal-03022935 \\ https://hal.uca.fr/hal-03022935}

Submitted on 25 Nov 2020

HAL is a multi-disciplinary open access archive for the deposit and dissemination of scientific research documents, whether they are published or not. The documents may come from teaching and research institutions in France or abroad, or from public or private research centers.
L'archive ouverte pluridisciplinaire HAL, est destinée au dépôt et à la diffusion de documents scientifiques de niveau recherche, publiés ou non, émanant des établissements d'enseignement et de recherche français ou étrangers, des laboratoires publics ou privés.

\section{(c)(1)}

Distributed under a Creative Commons Attribution| 4.0 International License 


\section{Optical calcium test for measurement of multiple permeation pathways in flexible organic optoelectronic encapsulation}

Patrick Boldrighini ${ }^{1}$, Aurélie Fauveau ${ }^{1}$, Sandrine Thérias ${ }^{2},{ }^{*}$ Jean-Luc Gardette ${ }^{2}$, Manuel Hidalgo ${ }^{3}$, *Stéphane $\operatorname{Cros}^{1}$

${ }^{1}$ CEA/LITEN/DTS/SMPV/LMPO, Institut National de I'Energie Solaire, 50 avenue du Lac Léman, 73377 Le Bourget du Lac, France

${ }^{2}$ Université Clermont Auvergne, Institut de Chimie de Clermont-Ferrand, UMR 6296 CNRS / UBP / Sigma Campus Universitaire des Cézeaux , 24 avenue Blaise Pascal , TSA 60026 - CS 60026, 63178 Aubière cedex, France

${ }^{3}$ ARKEMA. SOLLIA joint laboratory at Institut National de l'Energie Solaire, 50 avenue du Lac Léman, 73377 Le Bourget du Lac, France

Abstract

Organic photovoltaic (OPV) devices and other organic electronics have the promise to provide lightweight, flexible alternatives to traditional rigid semiconductor technologies. However, organic electronics often degrade rapidly upon exposure to oxygen, water, light and combinations thereof, as well as upon exposure to elevated temperatures. This requires the use of high gas barrier packaging in order for devices to have operational lifetimes on the order of years. To meet the challenge of transparent high gas barrier materials which maintain the flexibility of organic optoelectronics, many different materials and encapsulation schemes have been developed including the lamination of devices between flexible multi-layer barrier films. Because of their excellent barrier properties, these materials often require specialized testing for permeation measurements which evaluate materials independently. In this work, we demonstrate the use of an optical calcium test, which uses a sample geometry that closely mimics an OPV device, to evaluate a complete encapsulation scheme and to elucidate the relative importance of different permeation pathways. Using an encapsulation scheme of laminating a device between two multi-layer barrier films using an adhesive, measurements were made for water vapor permeation through the barrier film, the bulk adhesive, and along the adhesive-to-barrier film interface. The results show that the combined lateral permeation, including through the bulk adhesive and along

\footnotetext{
*Corresponding authors : Jean-Luc Gardette (luc.gardette@uca.fr) and Stéphane Cros (Stephane.CROS@cea.fr)
} 
the adhesive-to-barrier film interface, can constitute over $50 \%$ of the total permeation for small devices $(4.5 \mathrm{~cm} \times 4.5 \mathrm{~cm})$. The adhesive-to-barrier film interface was also found to be a very important pathway as it was deemed responsible for more permeation than the bulk adhesive. The technique was also used to evaluate encapsulation design variables such as the effects of adhesive thickness and surface treatments on the lateral water permeation. We demonstrate that decreasing the adhesive thickness leads to a decrease in the lateral water permeation.

\section{Introduction}

Organic optoelectronic devices, including organic photovoltaic devices (OPV) and organic light emitting diodes (OLED), have received much research attention due to their ability to provide flexible

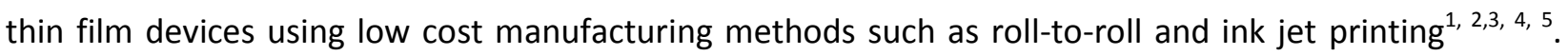
However, a significant hurdle to their widespread implementation still remains in the form of the sensitivity of these devices to water, oxygen, light and combinations thereof ${ }^{6,7,8}$. Previous studies have concluded that in order to achieve lifetimes of several years for OPV devices a water vapor transmission rate (WVTR) of $10^{-3} \mathrm{~g} / \mathrm{m}^{2} /$ day or less is needed and drops even lower to about $10^{-6} \mathrm{~g} / \mathrm{m}^{2} /$ day for OLEDs ${ }^{9}$. This sensitivity has spurred the research of transparent high barrier encapsulation materials and schemes that maintain the flexibility and low cost processing of the devices.

One popular approach is to laminate the device between two high barrier films using an adhesive to make the seal ${ }^{10,11}$. The permeation rates of the films used in this type of encapsulation can be evaluated in several different manners including permeation cells, electrical calcium tests and optical calcium tests ${ }^{12,13,14,15}$. Adhesives are generally impractical for measurement in permeation cells and instead often use simpler gravimetric techniques or optical calcium tests to measure their permeation rates $^{16,17}$. This leaves the optical calcium test as the only tool which is regularly used to measure both barrier films and adhesives, though the testing procedures differ greatly between the two methods. Optical calcium tests of flexible barrier films generally use high barrier edge seals to limit any side ingress of water vapor. On the other hand, tests for adhesives use glass substrates to block all permeation other than side ingress. Additionally, the use of glass substrates may cause any characterization of the lateral permeation to be inaccurate, as it does not have the same interfaces present in actual flexible encapsulation.

When using the scheme of laminating flexible barrier films with adhesives, there are several design factors to consider beyond the selection of materials. These include the distance from the edge of the device to the edge of the encapsulation, the thickness of the adhesive layer, and the processes used 
to apply the adhesive and laminate the device. These design factors are often tested using actual devices, though optical calcium tests have also been used to screen design choices for other encapsulation schemes ${ }^{18,11}$..

In this work, we present a modified version of the optical calcium test which uses a sample geometry that closely mimics a device and can evaluate both flexible barrier films and adhesives at the same time. This version of the optical calcium test also allows for the estimation of the relative importance of three different permeation pathways, including the adhesive-to-barrier film interface, thus allowing for the identification of any weak points in the encapsulation scheme. The ability to mimic a device also allowed this test to demonstrate the effects of encapsulation design including adhesive thickness.

\section{Experimental}

\subsection{Calcium Test Sample Preparation}

All samples were prepared to the dimensions seen in Figure 1. All flexible multi-layer barriers were VX 25T-2PO films from Oike \& Co., Ltd. (Japan). They were supplied with a removable protective film over the poly(ethylene terephthalate) (PET) surfaces of the barrier film. The barrier films were sonicated in isopropyl alcohol (IPA) for two cycles of $15 \mathrm{~min}$ after removal of the protective film to ensure a clean and consistent surface for bonding. Once dry, the films were laminated to the adhesive on one side. The adhesives used were the pressure sensitive adhesives (PSA) 467MP and 468MP, both from 3M (USA). These adhesives have the same chemical composition but different thicknesses of $50 \mu m$ and $125 \mu \mathrm{m}$ respectively. $5 \mathrm{~cm}$ X 5cm coupons of Dupont-Teijin Melinex 401 PET purchased from Micel Corp. (France) were cleaned via sonication in IPA for 2 cycles of $15 \mathrm{~min}$.

All laminations were performed using a weighted hand roller that met the requirements of PSTC Standard 101 Appendix B. Immediately after cleaning, the barrier films were laminated to the adhesive in air under ambient conditions. To remove any water vapor in the materials, the adhesive coated barrier films and PET coupons were then placed under vacuum (1-3mbar) at $80^{\circ} \mathrm{C}$ for 2 days before being allowed to cool to $30^{\circ} \mathrm{C}$ while still under vacuum. The adhesive coated barrier films were then quickly moved to a dry nitrogen glove box.

$45 \mathrm{~mm} \times 45 \mathrm{~mm} \times 160 \mathrm{~nm}$ deposits of $\geq 99.5 \%$ calcium from Cerac Inc. (USA) were deposited onto the PET coupons via evaporation under vacuum $\left(10^{-6} \mathrm{mbar}\right)$ at a rate of $<2 \mathrm{~nm} / \mathrm{s}$ using an evaporation chamber located inside a dry nitrogen glove box. The calcium deposition was measured using a quartz microbalance incorporated into the evaporation chamber. While remaining in the dry nitrogen glove 
box, the calcium coupons were then laminated between two adhesive coated barrier films. The laminated samples were then cut to $60 \mathrm{~mm} \times 60 \mathrm{~mm}$ with the calcium deposit in the center. All samples were stored in a dry nitrogen glove box for at least 3 days after lamination to allow the adhesive to achieve its full adhesion. Four samples were made per test condition and have been named using the system $X-Y$ where $X$ is the adhesive thickness in microns (100 or 250 ) and $Y$ is the sample number. For example, the first sample with a total adhesive thickness of $100 \mu \mathrm{m}$ would be named $100-1$.
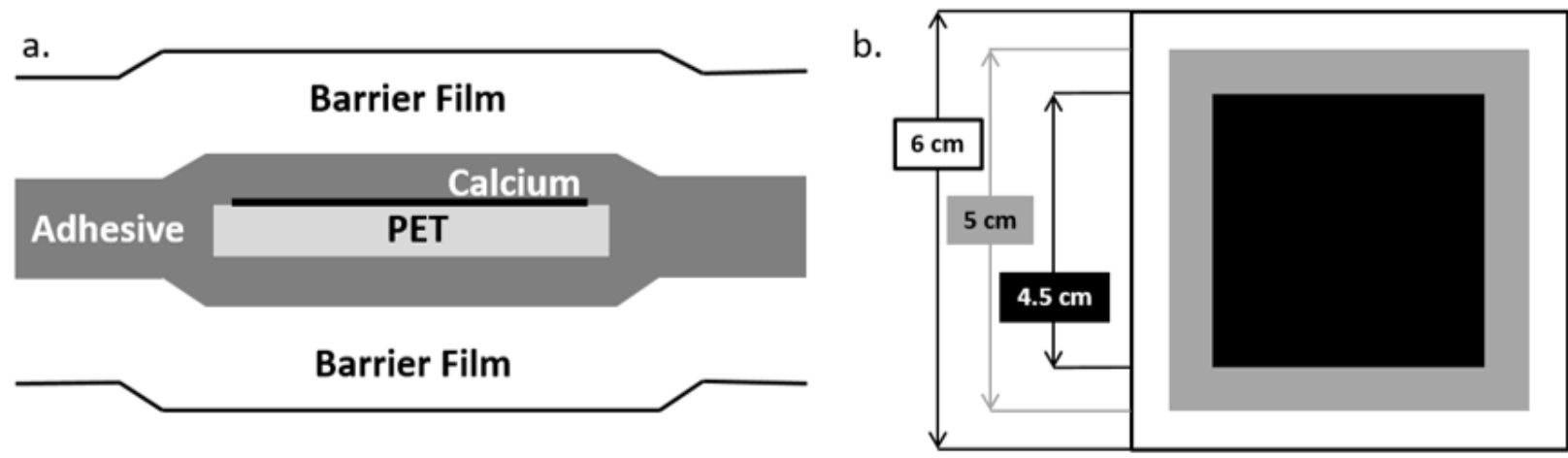

Figure 1

A diagram of the sample geometry for the proposed version of the optical calcium test is shown in (a) cross-sectional view and (b) top view. In the top view, the sample is a square and the marked dimensions are also the same for the horizontal dimensions. Please note that the diagram is not drawn to scale.

\subsection{Optical Calcium Test Configuration}

Images were taken in transmission using the configuration seen in Figure 2. The light source was a ML-0405 cold cathode fluorescent lamp from Coherent Inc. (USA). To limit the light seen by the camera, samples were placed into stainless steel holders that blocked all light except through a $5 \mathrm{~cm} \mathrm{X}$ $5 \mathrm{~cm}$ window in which the calcium deposit was centered. The camera was a DP70 Microscope Digital Camera from Olympus Optical Co., Ltd. (Japan) and was assembled using a U-TV1X-2 video port and a UCMAD3 C-mount camera adapter. Images were captured using DPController 1.2.1.108 and DPManager 1.2.1.107 software from Olympus Optical Co., Ltd. (Japan). The entire assembly, except for the computer, was located inside a light blocking enclosure in order to limit the effect of ambient light on the measurement. Images were taken periodically with storage of the samples between images in a humidity chamber at $65^{\circ} \mathrm{C}$ and $85 \%$ relative humidity. 


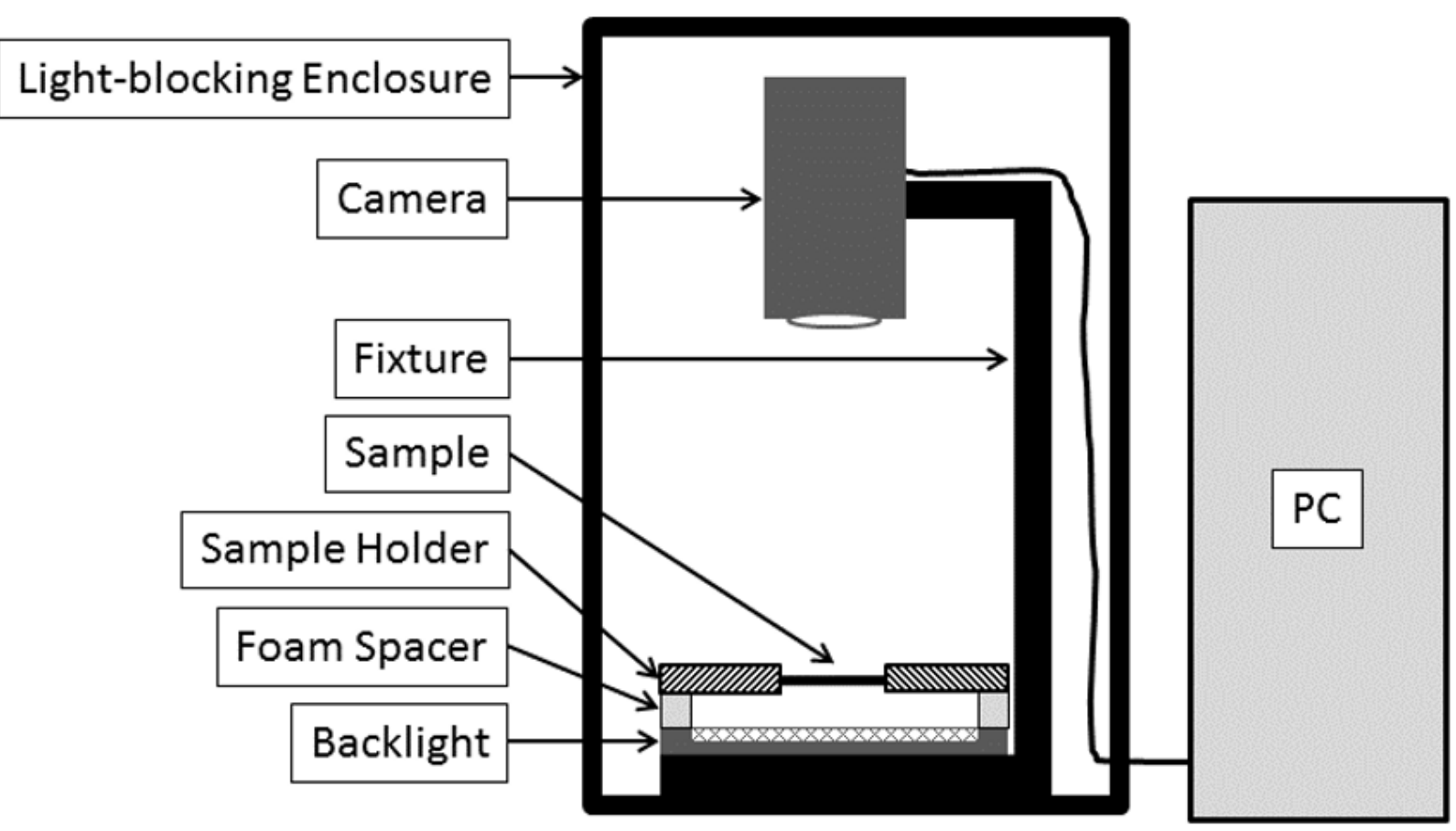

Figure 2

A cross-sectional diagram of the configuration used for imaging in the optical calcium test is shown with each component labelled.

\subsection{Image Analysis}

The grayscale values of each image were found using the open-source Java software ImageJ. Grayscale values were found as an average over the region being measured. The grayscale values are converted to a remaining calcium thickness using a calibration curve. The calibration curve is constructed by making gray scale measurements of samples with a known thickness of calcium. A curve of grayscale vs. calcium thickness can then be plotted. The calibration curve used in this study had a region of validity of $50-110 \mathrm{~nm}$ of calcium. Calcium layers outside this range of thicknesses were either too opaque or too transparent to measure accurately.

\subsection{Adhesion Testing}

T-peel adhesion tests were performed using a pull speed of $300 \mathrm{~mm} / \mathrm{min}$ on an Instron 3365 electromechanical load frame using a $50 \mathrm{~N}$ load cell. The sample width was $24 \mathrm{~mm}$ for all samples. The length of the samples was not controlled but was always greater than $12 \mathrm{~cm}$ and less than $18 \mathrm{~cm}$. The first and last $10 \%$ of the length of each sample was excluded from average adhesion strength calculations in 
order to avoid edge effects. All samples were prepared in the same manner as calcium test samples until removal from vacuum drying, at which point two adhesive coated barrier films were laminated together in air instead of being moved to the glove box.

\section{Results \& Analysis}

\subsection{Modified Optical Calcium Test}

The version of the optical calcium test used in this work is based upon the use of a sample geometry which closely mimics a device. This allows for results which closely approximate the permeation behavior that actual devices will experience, as there is a mix of permeation from several different pathways. These pathways include permeation orthogonal to the plane of the device across the barrier films and lateral permeation in the plane of the device. The lateral permeation is a mix of permeation in the bulk adhesive and along the adhesive-to-barrier film interface. In this sample geometry, the orthogonal permeation is seen as a gradual lightening of the calcium deposit as its thickness decreases. The lateral permeation is seen by the shrinking size of the metallic calcium as its edges are completely converted to transparent calcium hydroxide. As is seen in other lateral optical calcium tests, the lateral permeation creates a permeation front with the leading edge being at the edge of the metallic calcium. Therefore, it can be assumed that regions where metallic calcium is visible have not yet been subjected to water vapor which has permeated laterally. Because of this, a Central Zone that is subject to only orthogonal permeation, such as the one seen in Figure 3, can be defined over a region of calcium which is not affected by lateral permeation throughout the duration of the test. By analyzing the change in calcium thickness in this Central Zone, and thus the amount of permeated water, a measurement of the orthogonal permeation is possible.

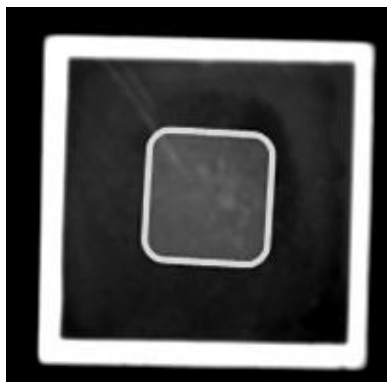

$\mathrm{O} \mathrm{hr}$

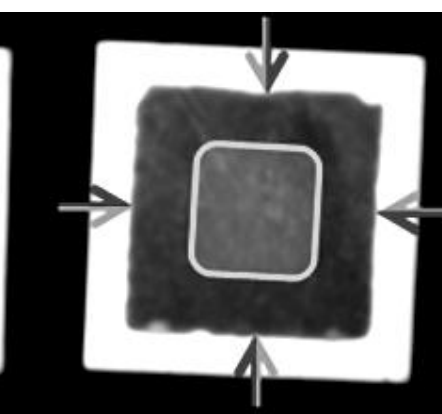

$24 \mathrm{hr}$

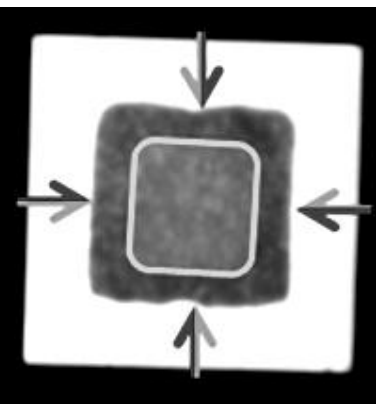

$48 \mathrm{hr}$

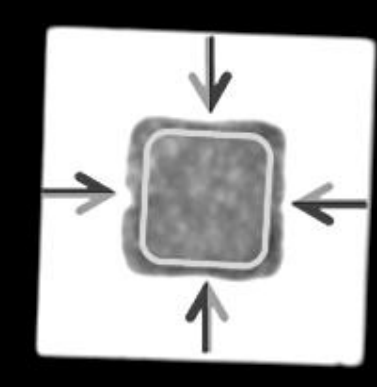

$72 \mathrm{hr}$

Figure 3 
A selection of images from optical calcium test sample 100-COR-3 is shown after various times of storage at $65^{\circ} \mathrm{C}$ and $85 \%$ relative humidity. The Central Zone remains unaffected by lateral permeation for the duration of the test.

An average gray scale for a Global Zone, which corresponds to the dimensions of the calcium deposit at the beginning of the test, is also found. Using the orthogonal permeation rates found in the Central Zone, the amount of calcium degradation due to orthogonal permeation for the entire Global Zone can be calculated. By subtracting this value from the total Global Zone degradation, the amount of calcium that has degraded due to lateral permeation, is found. This can also be converted to an apparent mass of permeated water, however this calculation greatly underestimates the actual amount of laterally permeated water, as it does not account for the non-zero concentration of water in the region behind the edge of the permeation front where all of the metallic calcium has already been degraded. This region has a concentration gradient with higher water vapor concentrations closer to the edge of the sample. Using simulations, Michels et al. have proposed that the exact shape of this concentration gradient is dependent on several factors and can vary from sample to sample ${ }^{17}$. For this reason it is impossible to estimate the amount of water in the region behind the edge of the permeation front unless the calcium test data is combined with other permeation testing and computer simulations. Although the value of apparent laterally permeated water calculated from the calcium test does not provide an accurate quantitative value, it is still quite useful for relative comparisons of different adhesives, edge seals and adhesive-to-barrier film interfaces.

When the mass of laterally permeated water is plotted against time, three unique regions are visible as seen in Figure 4. At the beginning in Region I, little to no degradation is observed as the leading edge of the permeation front has not reached the edge of the calcium. In Region II, the curve then becomes fairly linear, though not perfectly so. In Region III the curve then begins to flatten and tend towards a constant value. It is not uncommon for individual samples to actually display a slightly negative permeation rate at the end of the test. The behavior in Region III can be attributed to testing artifacts. Small voids often form as the calcium degrades and they expand as more water permeates into the adhesive. At the end of a test there is often enough water to reflect and refract enough light to produce a non-white gray scale value in transmission measurements. This apparent darkening of the already fully degraded areas is seen as the flattening of the water permeation curves. Therefore, all calculations for lateral permeation are limited to the region after the permeation front has reached the 
edge of the calcium but before $60 \%$ of the calcium deposit has been degraded, which corresponds to Region II in Figure 4.

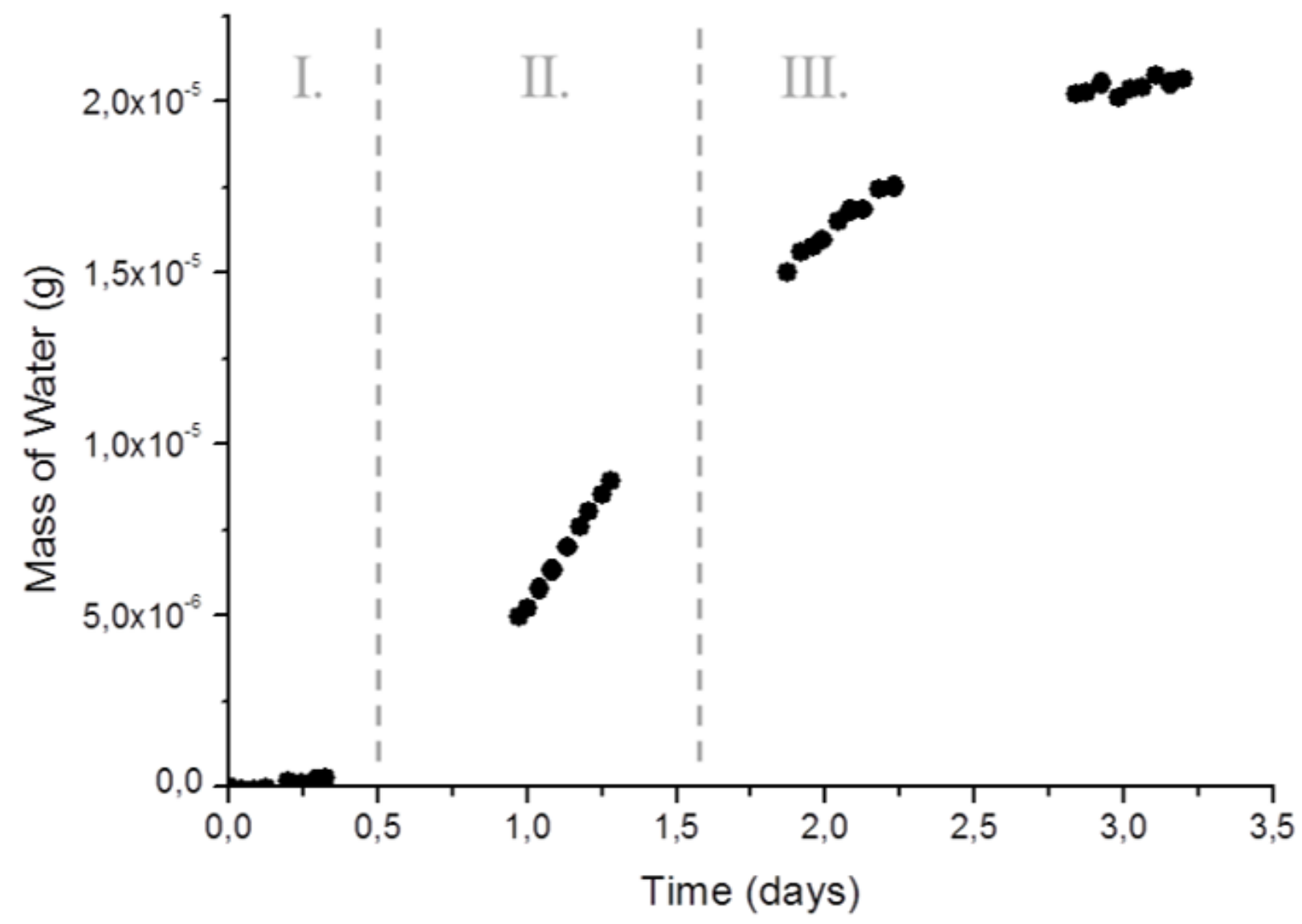

Figure 4

The cumulative lateral permeation as measured by the modified optical calcium test is shown for sample 100-3. Three distinct regions are visible. Region I shows little permeation as the permeation front has yet to reach the edge of the calcium deposit and Region III is invalid due to testing artifacts. Therefore only Region II is used for calculations.

This sample geometry also addresses the issue of interfacial permeation by incorporating the same adhesive-to-barrier film interface present in actual encapsulated devices. To separate the relative permeation in the bulk adhesive and the interface using the calculated values of apparent lateral permeation, the following three assumptions have been made in order to simplify the modeling:

1. Permeation in the bulk adhesive is not affected by changes in the interface.

2. Permeation along the interface is not affected by changes in the adhesive thickness.

3. Permeation in the bulk adhesive and along the interface are independent of each other. 
By assuming that the two pathways are independent of each other, the total lateral permeation becomes a summation of the two pathways. The permeation flux in bulk materials, $J$, is generally a function of the exposed surface area, which in the case of this sample geometry is the product of the perimeter, $p$, and the adhesive thickness, $h_{a d h}$. The interface, however, is essentially 2-D in nature with no effective thickness, thus making the interfacial permeation a function of the exposed perimeter of the interface. The summation of the pathways then becomes the following equation where $Q_{\text {lat }}$ is the mass of laterally permeated water, $J_{a p-a d h}$ is the apparent water vapor flux of the bulk adhesive in units of mass per time per area, and $\dot{P}_{\text {intf }}$ is the interfacial permeation rate in units of mass per time per length.

$$
\partial Q_{\text {lat }} / \partial t=p h_{a d h} J_{a p-a d h}+2 p \dot{P}_{\text {intf }}
$$

Experimentally, all values other than $J_{a p-a d h}$ and $\dot{P}_{\text {intf }}$ can be measured for a single sample. Using the assumption that changing the adhesive thickness will not impact the interfacial permeation, two samples with the same adhesive and interfaces but different adhesive thicknesses would be able to provide the information needed to calculate $J_{a p-a d h}$ and $\dot{P}_{\text {intf, }}$ and therefore also estimate the relative contributions of the bulk adhesive and the interface to the lateral permeation. As previously discussed for the apparent lateral permeation, $J_{a p-a d h}$ and $\dot{P}_{\text {intf }}$ are not accurate values and greatly underestimate the amount of permeation. Also, the apparent permeation flux of the bulk adhesive, while providing a good fit to the experimental data when considered to be constant, is an estimation of transient state permeation and should not be confused for a steady state WVTR. However, these values do provide the ability to elucidate information about the relative contributions of the bulk adhesive and the interface to the lateral permeation which in turn allows for a better understanding of where the weak points in the encapsulation lay.

\subsection{Comparison of Permeation Pathways}

The permeation in the Central Zone of each sample can be seen in Figure 5. For samples with $100 \mu \mathrm{m}$ of adhesive, the average orthogonal permeation was measured to be $3.89 \times 10^{-3} \mathrm{~g} / \mathrm{m}^{2} /$ day. Samples with $250 \mu \mathrm{m}$ of adhesive showed a slightly lower average orthogonal permeation of $2.46 \times 10^{-3}$ $\mathrm{g} / \mathrm{m}^{2} /$ day. These values represent the steady state permeation rate of water vapor in the orthogonal direction per unit area of exposed barrier film at $65^{\circ} \mathrm{C}$ and $85 \%$ relative humidity. 


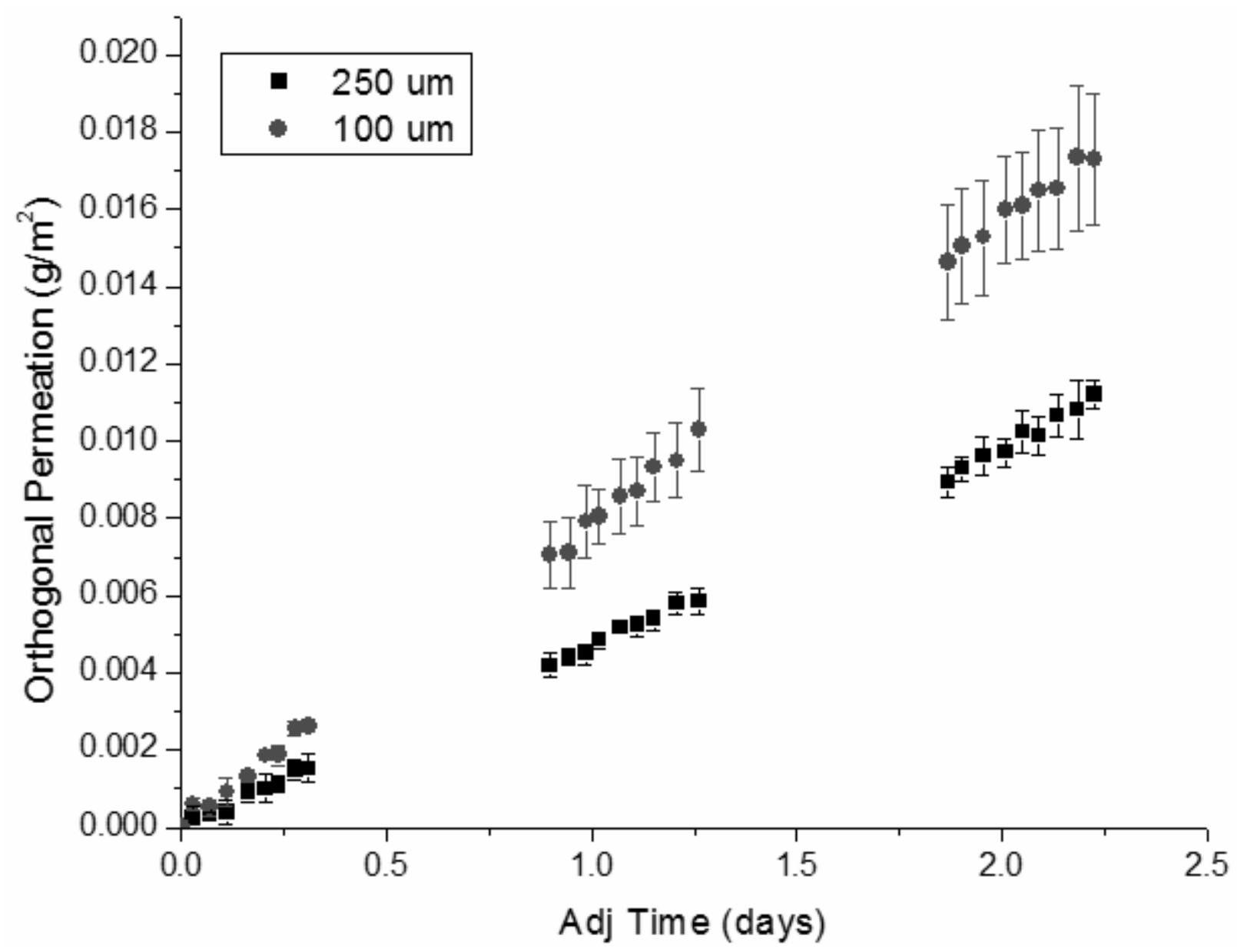

Figure 5

The cumulative water vapor permeation for each sample as measured by the optical calcium test is shown in units of mass of permeated water per unit area. The time axis has been adjusted to remove the effect of residual water in the samples reacting with the calcium at the start of the test. All measures were in the Central Zone of the respective samples and are linear which indicates steady state permeation.

The lateral permeation of each sample can be seen in Figure 6 . It can be clearly seen that samples with $250 \mu \mathrm{m}$ of adhesive had more lateral permeation than those with $100 \mu \mathrm{m}$ of adhesive. This is expected as the thicker adhesive layer has a larger surface area of adhesive exposed to the atmosphere. This is an important result as it directly influences the design of organic optoelectronic encapsulation. The experimental data from this study indicates that a thinner adhesive layer is indeed preferred for minimizing permeation. This information must be taken into account when weighing the benefits of a thinner adhesive layer (less permeation, thinner final product, less material used) against 
the benefits of a thicker adhesive layer (better adhesion strength, easier to avoid voids around the edges of the device).

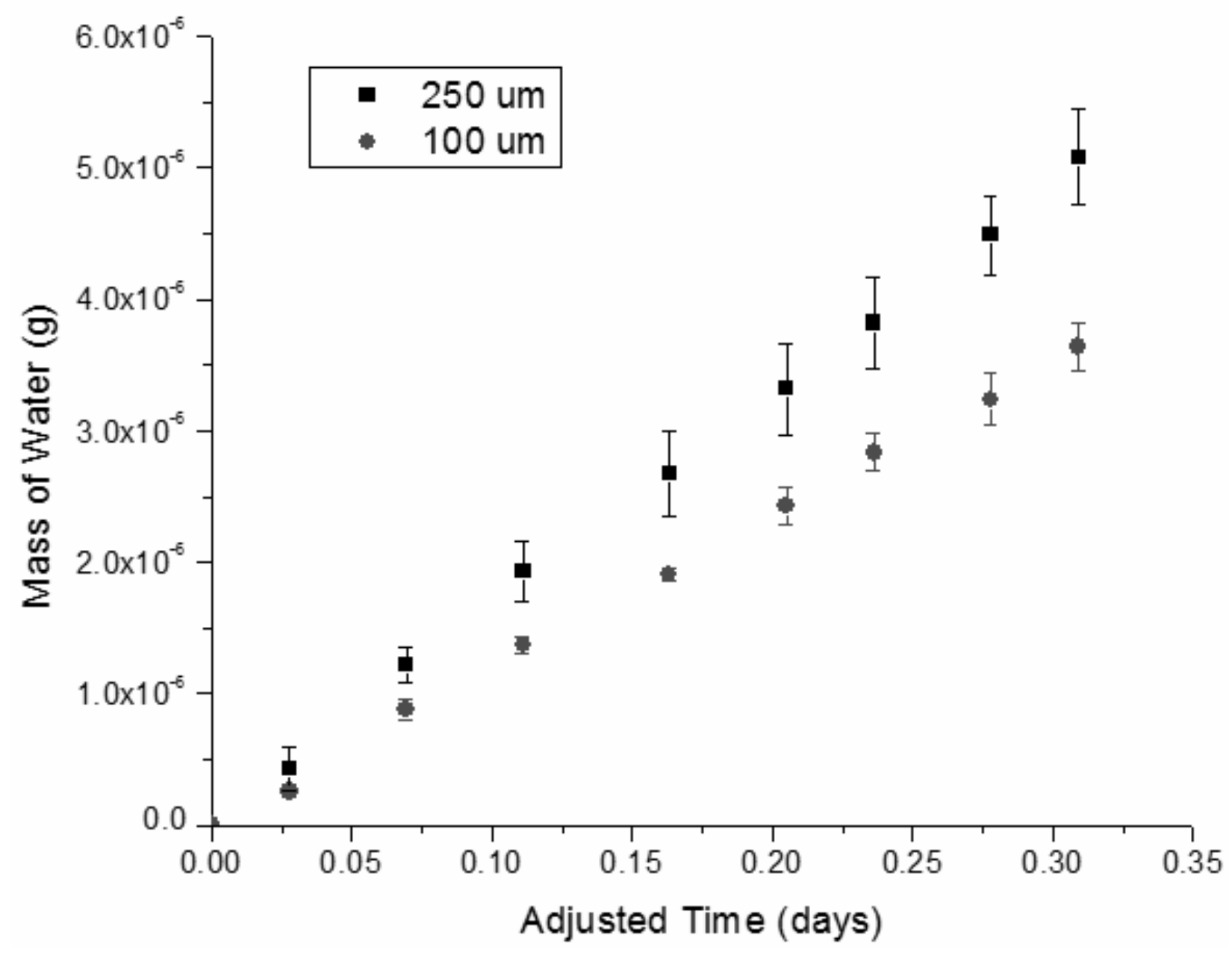

Figure 6

The cumulative lateral permeation as measured by the optical calcium test is shown for all samples. The time axis has been adjusted so that 0 corresponds to the first point after the permeation front had reached the edge of the calcium deposit.

Using the analysis method described earlier in this article, the relative impact of the barrier films, bulk adhesive and adhesive-to-barrier film interface were calculated. The average percentage of the calcium degraded by pathway is shown for each test condition in Table 1. This data clearly shows that lateral permeation accounted for a large proportion of the calcium degradation. This indicates the 
importance of limiting lateral permeation in organic optoelectronic packaging. This is especially true for smaller devices where there is less distance between the center of the device and the edge.

Another important result seen in Table 1 is the role of interfacial permeation compared to the bulk adhesive. The analysis described in this article estimates that more than half of the lateral permeation is due to the interface for all samples. This is despite the fact that adhesive does have good adhesion to the barrier film, indicating a strong interface. This high level of interfacial permeation demonstrates the need for this permeation pathway to be taken into account when designing encapsulation and why permeation testing with the same interfaces as an encapsulated device is a useful tool.

\begin{tabular}{|l|c|c|c|c|}
\cline { 2 - 5 } \multicolumn{1}{c|}{} & \multicolumn{4}{c|}{ Percentage of total calcium degradation } \\
\hline Sample & Orthogonal & Total Lateral & Bulk Adhesive & Interface \\
\hline $\mathbf{2 5 0} \boldsymbol{\mu m}$ & $\mathbf{3 8 \%}$ & $\mathbf{6 2} \%$ & $27 \%$ & $35 \%$ \\
\hline $\mathbf{1 0 0} \boldsymbol{\mu m}$ & $\mathbf{5 4 \%}$ & $\mathbf{4 6 \%}$ & $11 \%$ & $35 \%$ \\
\hline
\end{tabular}

Table 1

The average percentages of the total calcium degradation measured during optical calcium tests of each testing condition are shown for each permeation pathway.

\section{Conclusion}

The optical calcium test is an established and versatile testing tool for the permeation of water vapor and has been presented here as a technique that can closely simulate the permeation behavior seen in encapsulated organic optoelectronic devices. The presented analysis method allows for the simultaneous measurement of flexible barrier films and the estimation of lateral permeation. The respective amounts of lateral permeation coming from the bulk adhesive and the interface can also be estimated using this technique, thus allowing for a comparison of multiple permeation pathways. The ability to characterize the interface as a permeation pathway provides a unique advantage to this method of the optical calcium test despite the fact that the characterization of the lateral permeation is only qualitative in nature.

This optical calcium test method then demonstrated that lateral permeation can be a very important permeation pathway for small devices. An increase in the adhesive thickness was also shown to increase the amount of lateral permeation and must therefore be carefully considered when 
designing encapsulation for sensitive devices. Finally the impact of the interface on lateral permeation was found to be larger than that of the bulk adhesive for this system. This finding shows the importance of considering the adhesive-to-barrier film interface when evaluating the suitability of encapsulation materials, as this effect would not be found when evaluating the materials individually.

\section{Acknowledgements}

Financing for this work was provided by the Région Auvergne (France).

${ }^{1}$ C. N. Hoth, P. Schilinsky, S. A. Choulis, and C. J. Brabec, Nano Lett. 8(9) 2806 (2008) DOI: $10.1021 / \mathrm{nl} 801365 \mathrm{k}$

${ }^{2}$ F.C. Krebs, Sol. Energy Mater. Sol. Cells 93, 394 (2009).

${ }^{3}$ A.C. Arias, J. D. MacKenzie, I. McCulloch, J. Rivnay, and A. Salleo, Chem. Rev. 110 (1), 3 (2010)

${ }^{4}$ R.R. Sondergaard, M. Hosel, and F.C. Krebs,. Polym. Sci. Part B Polym. Phys. 51, 16 (2013).

${ }^{5}$ A. Teichler, J. Perelaer, and U. S. Schubert, J. Mater. Chem. C. 1, 1910 (2013)

${ }^{6}$ M. Jørgensen, K. Norrman, and F. C. Krebs Solar Energy Materials \& Solar Cells 92, 686 (2008)

${ }^{7}$ M. Jørgensen, K. Norrman, S.A.Gevorgyan, T. Tromholt, B. Andreasen, and F.C. Krebs, Adv Mater. 2, 580 (2012).

${ }^{8}$ X. Wang, Z. Xinxin, C., G. Xu, Z.K. Chen, and F. Zhu, Sol. Energy Mater. Sol. Cells 104, 1 (2012).

${ }^{9}$ S. Cros, R. de Bettignies, S. Berson, S. Bailly, P. Maisse, N. Lemaitre, and S. Guillerez, Sol. Energy Mater. Sol. Cells 95, S65 (2011).

${ }^{10}$ H. Klumies, M. Karl, M. Hermenau, R. Rösch, M. Seeland, H. Hoppe, L.Müller-Meskamp, and K. Leo, Solar Energy Materials \& Solar Cells 120, 685 (2014)

11 J. Ahmad, K. Bazaka, L.J. Anderson, R.D. White, and M.V. Jacob, Renew. Sustain. Energy Rev. 27, 104 (2013).

12 R. Paetzold, A. Winnacker, D. Henseler, V. Cesari, V. and K. Heuser, Rev. Sci. Instrum. 74, 5147 (2003).

${ }^{13}$ M.O. Reese, A. Dameron, and M.D. Kempe,. Rev. Sci. Instrum. 82, 085101 (2011).

${ }^{14}$ G. Nisato, P. C. P. Bouten, P. J. Slikkerveer, W. D. Bennett, G. L. Graff, N. Rutherford, and L. Wiese, in Proceedings of Asia Display, 8th IDW (Soc. Inform. Display, San Jose, CA, 2001), p. 1435

${ }^{15}$ H. Klumbies, L. Müller-Meskamp, T. Mönch, S. Schubert, and K. Leo, Rev. Sci. Instrum. 84, 024103 (2013).

${ }^{16}$ T. Braun, F. Hausel, J. Bauer, O. Wittler, R. Mrossko, M. Bouazza, K.-F. Becker, U. Oestermann, M. Koch, V. Bader, C. Minge, R. Aschenbrenner, and H. Reichl, Conference Paper in Proceedings - Electronic Components and Technology Conference · June 2008 DOI:10.1109/ECTC.2008.4549970

${ }^{17}$ J.J. Michels, J. J., M. Peter, A. Salem, B. van Remoortere, and J. van den Brand, J. Mater. Chem. C. 2, 5759 (2014).

18 J. Granstrom, M. Gällstedt, M. Villet, J.S. Moon, and T. Chatterjee, Thin Solid Films 518, 5282 (2010). 